\title{
Select Line Speeds for Single-Hub SONET/WDM Ring Networks
}

\author{
Xiang-Yang Li, Peng-Jun Wan
}

\begin{abstract}
Minimizing SONET ADM costs in single-hub SONET/WDM ring networks via traffic grooming has been discussed in a number of recent works. Recent work [12] gives the exact minimum costs of uniform traffic in both UPSR and BLSR/2 and proves that the BLSR/2 would never be more expensive than UPSR under any traffic pattern, if all wavelengths have same capacity.

In this paper we consider how to groom both uniform and non-uniform traffic to minimize the cost of ADMs in the single-hub UPSR and BLSR/2 with mixed line speeds. We especially explore the grooming of traffic when wavelengths have two different capacities $g_{1}=1$ and $g_{2}=4$. We show that the problem can be confined to just consider the traffic request $r_{i} \leq 4$ for all non-hub node $i$. By adopting the same cost model as in [5], i.e., ADMs with speed $g_{1}=1$ and $g_{2}=4$ cost 1 and 2.5 respectively, we provide optimal traffic partition and grooming for uniform traffic demands, and develop optimal or suboptimal solutions for non-uniform traffic demands, depending on the range of all demands from non-hub nodes.
\end{abstract}

\section{INTRODUCTION}

Coupling wavelength division multiplexed (WDM) technology [9] with synchronous optical network (SONET) rings [6] can not only greatly increase capacity, thereby reducing the amount of required fiber and allowing for more graceful upgrades, but also potentially reduce the amount of required SONET terminal equipment, the SONET Add/Drop Multiplexers (ADMs), by allowing individual wavelengths to optically bypass a node via a wavelength add-drop multiplexer (WADM) rather than being electronically terminated [3]. Typically, the traffic demand between two nodes is low rated (e.g., $O C-3$ ), and a high-rate (e.g., $O C$-48) SONET ring can carry a number of such low-speed traffic streams. With WADM, the number of ADMs required in a SONET ring is equal to the number of nodes that are endpoints of some requests carried in this ring. Thus the optimal grooming problem is to partition the set of communication requests into a number of groups such that each group can be carried in a single SONET ring and the total ADM cost is minimized. The minimum ADM cost depends on both the underlying network architecture and the traffic pattern. Three types of SONET self-healing rings have been defined by standard bodies [6]: a unidirectional path-switched ring (UPSR); a two-fiber bidirectional line-switched ring (BLSR/2); a four-fiber bidirectional line-switched ring (BLSR/4).

The SONET self-healing rings are employed in both access networks and in inter-office networks. In access networks, the traffic streams between access nodes are routed by going through the telephone company's central office. In order to increase the channel utilization, a digital cross-connect is often installed in the central office to cross connect the traffic streams.

\footnotetext{
Department of Computer Science, University of Illinois at UrbanaChampaign, Urbana, IL 61801. Email: xli2@cs.uiuc.edu. Part of the work was done, when the author visited the Argonne National Laboratory.

Department of Computer Science, Illinois Institute of Technology, Chicago, IL 60616. Email: wan@cs.iit.edu.
}

The central office equipped with a digital cross-connect is referred to as a hub and the SONET ring with a hub is referred to as a single-hub ring.

In addition to the network architectures, the minimum ADM cost also varies upon the traffic pattern and traffic demands. The traffic could have some regular patterns such as one-to-all and all-to-all, or any irregular pattern. The traffic demands may be uniform (i.e. all traffic have the same amount of demands) or non-uniform. Each traffic demand itself is given as an integer number of low speed (tributary) streams. Alternatively, it can also be represented by its traffic granularity, defined as the ratio of its demand to the transmission capacity of a single wavelength. A traffic is said to be a full-wavelength traffic, a sub-wavelength traffic or a super-wavelength traffic if its traffic granularity is equal to one, greater than one, or less than one respectively.

The minimum ADM problem has been discussed in a number of recent works [2] [4] [5] [7] [8] [10] [11]. [4] and [7] studied optimal grooming of arbitrary full-wavelength lightpaths. [2], [10] and [11] provided grooming of uniform $\frac{1}{2}, \frac{1}{4}$, and $\frac{1}{8}-$ wavelength traffic. [5] and [8] gave some preliminary results on the traffic grooming in single-hub rings. In [8], an optimal grooming of uniform one-to-all sub-wavelength traffic in singlehub UPSR rings was presented. [5] briefly discusses the criteria for using UPSR vs. BLSR rings and to mix two types of line speeds on a single SONET/WDM ring. In [12], the authors further the works in [5] and [8] and provide stronger results about the ADM cost of uniform all-to-all traffic in both single-hub UPSR and single-hub BLSR/2. They establish a reduction from grooming of any duplex traffic to grooming of one-to-all duplex traffic, and from grooming of one-to-all duplex traffic to grooming of one-to-all simplex traffic. Thus any optimal grooming of one-to-all simplex leads to an optimal grooming of one-to-all duplex and an optimal grooming of all-to-all duplex. Therefore, from then on we concentrate on only one-to-all simplex traffic. They also show that BLSR/2 always costs no more than UPSR under any traffic and the search for optimal grooming can be confined to a narrow subset of valid groomings, referred to as canonical groomings. They then construct optimal canonical groomings of uniform one-to-all traffic in both UPSR and BLSR/2 rings and derive the analytic expression of the minimum ADMs.

The paper is structured as follows. We review the results in[12] for optimal traffic grooming in single-hub SONET/WDM rings with only one line speed in Section II. It was proved [12] that the search of optimal grooming of uniform traffic in UPSR and BLSR/2 can be confined to those canonical groomings. We analyze the basic properties of arbitrary traffic grooming when wavelengths have two different capacities $g_{1}=1$ and $g_{2}=4$ and the cost of corresponding ADMs is 1 and 2.5 respectively 
in Section III. And we show that the problem can be confined to just consider the traffic request $r_{i}<4$.for all non-hub node $i$ In Section IV and Section V, we provide optimal traffic partition and grooming for uniform traffic demands, and develop optimal or suboptimal solutions for non-uniform traffic demands, depending on the range of all demands from non-hub nodes. Finally we conclude our paper in section VI.

\section{PRELIMINARIES}

We consider a single-hub SONET/WDM ring comprising of $n+1$ nodes numbered $0,1, \cdots, n$,clockwise, with the hub placed at node 0 . The traffic demand and the transmission capacity of each wavelength are in terms of the basic low-rate (e.g., $O C-3)$ traffic streams. We first review the result in [12] for optimal traffic grooming in single-hub SONET/WDM rings with only one line speed. Let $g$ be the transmission capacity of a single wavelength.

In [8], it was proved that the search of optimal grooming of uniform sub-wavelength traffic in UPSR can be confined to those groomings satisfying that each demand is carried in exactly one wavelength, i.e., split of a demand into more than one wavelengths is not allowed. In [12], the property is generalized to arbitrary traffic pattern with arbitrary traffic demands in both UPSR and BLSR/2.

Given a set of demands $\left\{r_{1}, \cdots, r_{n}\right\}$ in a UPSR and the wavelength capacity $g$, a grooming is said to be a canonical grooming if at each node $1 \leq i \leq n$, its demand is carried in $\left\lceil\frac{r_{i}}{g}\right\rceil$ wavelengths, among which $\left\lfloor\frac{r_{i}}{g}\right\rfloor$ wavelengths each carries $g$ units of demands to node $i$, and the remaining one, if there is any, carries $r_{i} \bmod g$ units of demands to node $i$.

Given a set of demands $\left\{r_{1}, \cdots, r_{n}\right\}$ in a BLSR/2 and the wavelength capacity $g$, a grooming is said to be a canonical grooming if at each node $1 \leq i \leq n$, its demand is carried in $\left\lceil\frac{r_{i}}{\frac{g}{2}}\right\rceil=\left\lceil\frac{2 r_{i}}{g}\right\rceil$ wavelengths (counting each wavelength used in both directions as two), among which $\left\lfloor\frac{r_{i}}{\frac{g}{2}}\right\rfloor=\left\lfloor\frac{2 r_{i}}{g}\right\rfloor$ wavelengths each carries $\frac{g}{2}$ units of demands to node $i$, and the remaining one, if there is any, carries $r_{i} \bmod \frac{g}{2}$ units of demands to node $i$.

The next lemma states that when looking for optimal traffic grooming for single-hub SONET/WDM rings with single line speed, we can pay attention to only canonical groomings.

Lemma 1: [12] Given any set of demands in UPSR or $\mathrm{BLSR} / 2$, there is a canonical grooming with minimum ADM cost.

In this section, we present optimal grooming of uniform traffic in both single-hub UPSR and single-hub BLSR/2. We assume that the traffic demand from the hub to each other node is $r$. [8] essentially gave the optimal canonical grooming in single-hub UPSR when $r<g$. In [12], they present the optimal canonical grooming for arbitrary $r$ in both single-hub UPSR and single-hub BLSR/2. For completeness, we give a review of their approach to construct the optimal canonical grooming.
Let's first consider the optimal grooming of uniform traffic in single-hub UPSR.

If $r \bmod g=0$, then the optimal canonical grooming is unique in the sense that each wavelength carry $g$ units of demands exclusively to some node. Thus each node contributes $2 \cdot \frac{r}{g}=\frac{2 r}{g}$ ADMs, half at the node itself and half at the hub. So the total ADM cost in the working fiber is $n \cdot \frac{2 r}{g}=\frac{2 n r}{g}$. The total ADM cost is then $\frac{4 n r}{g}$.

Now we assume that $r \bmod g>0$. In any canonical grooming, at each node there are $r-r \bmod g$ portion of demands carried in $\left\lfloor\frac{r}{g}\right\rfloor$ wavelengths exclusively. These demands use $2 n\left\lfloor\frac{r}{g}\right\rfloor$ ADMs in the working fiber. In any optimal grooming, the remaining demands at each node, referred to as residue demands, must use a minimum ADM cost. This can be achieved in the same way as in [8]. We partition the $N$ nodes into

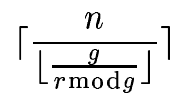

groups of at most $\left\lfloor\frac{g}{r \bmod g}\right\rfloor$ nodes. The residue demands of nodes in each group are carried in a single wavelength. These residue demands totally require

$$
n+\left\lceil\frac{n}{\left\lfloor\frac{g}{r \bmod g}\right\rfloor}\right.
$$

ADMs in the working fiber. Thus the total ADMs used in the working fiber is

$$
\begin{aligned}
& 2 n\left\lfloor\frac{r}{g}\right\rfloor+n+\left\lceil\frac{n}{\left\lfloor\frac{g}{r \bmod g}\right\rfloor}\right\rceil \\
& =n\left\lceil\frac{r}{g}\right\rceil+n\left\lfloor\frac{r}{g}\right\rfloor+\left\lceil\frac{n}{\left\lfloor\frac{g}{r \bmod g}\right\rfloor}\right\rceil .
\end{aligned}
$$

Let

$$
F(g, r, n)=\left\{\begin{array}{cc}
\frac{2 n r}{g} & \text { If } r \bmod g=0, \\
n\left\lceil\frac{r}{g}\right\rceil+n\left\lfloor\frac{r}{g}\right\rfloor^{2}+\left\lceil\frac{n}{\left\lfloor\frac{g}{r \bmod g}\right\rfloor}\right\rceil & \text { otherwise. }
\end{array}\right.
$$

Then the minimum ADM cost in the working fiber is $F(g, r, n)$, and the total ADM cost is $2 F(g, r, n)$.

Similarly, the minimum ADM cost in BLSR/2 is $F\left(\frac{g}{2}, r, n\right)$. The optimum canonical grooming can be constructed in the similar way.

The next theorem summarizes the above discussions.

Theorem 2: The minimum ADM cost of uniform traffic demand with rate $r$ in UPSR and BLSR/2 is $2 F(g, r, n)$ and $F\left(\frac{g}{2}, r, n\right)$ respectively.

\section{Select Speeds with two Line SpeEds Available}

In the previous section, we assume that all SONET rings have the same line speed. In this case, the higher the line speed, the smaller the number of ADMs. On the other hand, the higher the line speed, the higher the cost of the ADM. However, the cost of ADM does not increase linearly with the line speed. The cost model adopted in [5] assumes that the cost ratio between an $O C-4 n \mathrm{ADM}$ and an $O C-n \mathrm{ADM}$ is 2.5 . If the traffic demand is 
uniform, then the best line speed can be selected by comparing the total ADM cost for each line speed.

However, if we allow the SONET rings to have different line speeds, we have to partition the traffic from each node into the SONET rings of different line speeds. After the partition, the traffic grooming algorithms developed in the previous sections can be applied to the rings of any particular line speed. Thus a solution has two components, the partition of the traffic, and the groomings of the traffic in rings of each speed. Both components affect the overall cost. Because there are a very large number of possible traffic partitions, it's impossible to find the best solution by enumeration. This is true even if all traffic demands are uniform. So efficient algorithms or criteria should be developed to find traffic partitions which may lead to the minimum ADM cost. This section is intended to address this problem.

To simplify the problem, we assume that there are only two line speeds $g_{1}$ and $g_{2}$ with $g_{2}=4 g_{1}$ as did in [5]. We also adopt the same cost model used in [5]. We assume that the cost of a ADM of speed $g_{1}$ is one, and the cost of a ADM of speed $g_{2}$ is 2.5. A simple approach presented in [5] is that for each traffic demand with value $r$, assign $r \bmod g_{2}$ traffic to the SONET rings with speed $g_{1}$ and $r-r \bmod g_{2}$ traffic to the SONET rings with speed $g_{2}$. The performance of this approach comparing to the optimal assignment was not discussed in [5]. In this section, more general solutions will be developed and their optimality will also be proven. In particular, a complete optimal solution for uniform traffic demands is obtained.

\section{A. Basic Properties}

As there are only two type of speeds, we call a SONET ring of speed $g_{1}$ as a low-speed ring, and a SONET ring of speed $g_{2}$ as a high-speed ring without any ambiguity. Similarly, we call a SONET ADM of speed $g_{1}$ as a low-speed ADM, and a SONET ADM of speed $g_{2}$ as a high-speed ADM. For the simplicity of presentation, $g_{1}$ is scaled to one and all demands are scaled accordingly. Thus $g_{1}=1, g_{2}=4$ and all demands are fractional numbers or integers.

In this section, we will study the selection of line speed in UPSR in detail. The analysis can be extended to BLSR as well. Because the ADM cost of the working ring is exactly the same as the protection ring, we can only consider the cost of the working ring. Assume the demand between node $i$ and hub is $r_{i}$ for $1 \leq$ $i \leq n$. Then any traffic partition can be represented by an $n$ dimensional vector

$$
f=\left(f_{1}, \cdots, f_{n}\right)
$$

where $0 \leq f_{i} \leq r_{i}$ is the amount of the traffic between node $i$ and hub placed to a low-speed ring. For any traffic partition, we can groom the traffic carried in low-speed rings and the traffic carried in high-speed rings separately. If both the grooming of the traffic carried in low-speed rings and the grooming of the traffic carried in high-speed rings are canonical, we call the overall grooming is canonical too.
In the following, we will present some basic properties of optimal traffic partitions.

Lemma 3: In any optimal traffic partition $f=\left(f_{1}, \cdots, f_{n}\right)$, $f_{i}<3$ for all $1 \leq i \leq n$, and there is an optimal solution $f=\left(f_{1}, \cdots, f_{n}\right)$ with $f_{i} \leq 2$ for all $1 \leq i \leq n$.

Proof: We prove the first part of lemma by contradiction. Let $f=\left(f_{1}, \cdots, f_{n}\right)$ be any optimal traffic partition with $f_{i} \geq 3$. Then in a canonical optimal grooming, there at least three low-speed rings devoted exclusively to node $i$. If we move the traffic carried in any three of these low-speed rings into one-speed ring, we save 6 low-speed ADMs and uses two new high-speed ADMs, and thus decrease the cost by 1 . This contradicts to the optimality of $f=\left(f_{1}, \cdots, f_{n}\right)$. We now prove the second part of lemma by contradiction. Let $f=\left(f_{1}, \cdots, f_{n}\right)$ be any optimal traffic partition which contains the least number of entries that are more than two. Suppose $f_{i}>2$ for some $1 \leq i \leq n$. Then in a canonical optimal grooming of the traffic demands $\left\{f_{1}, \cdots, f_{n}\right\}$ into low-speed rings, at least $\left\lceil f_{i}\right\rceil+\left\lfloor f_{i}\right\rfloor$ ADMs are devoted to node $i$. Now we place such $f_{i}$ amount of traffic from node $i$ into $\left\lceil\frac{f_{i}}{4}\right\rceil$ new high-speed rings, i.e. set $f_{i}=0$. Then in the new solution, a cost of at least $\left\lceil f_{i}\right\rceil+\left\lfloor f_{i}\right\rfloor$ is saved from the rings of speed $g_{1}$ while a cost of $5\left\lceil\frac{f_{i}}{4}\right\rceil$ is added to the rings of speed $g_{2}$. As

$$
\left\lceil f_{i}\right\rceil+\left\lfloor f_{i}\right\rfloor \geq 5\left\lceil\frac{f_{i}}{4}\right\rceil
$$

when $f_{i}>2$, the new solution has no more cost than the solution $f$ but contains one less entries which are more than two. This contradicts to the selection of $f$. Therefore, the lemma is true.

Intuitively, if a traffic can fill a high-speed ring, it should fill fully as many high-speed rings as possible to take advantage of the lower cost per bandwidth of the higher speed ring. The next lemma verifies such intuition.

Lemma 4: There is an optimal traffic partition $f=$ $\left(f_{1}, \cdots, f_{n}\right)$ with $f_{i} \leq r_{i} \bmod 4$ for all $1 \leq i \leq n$.

Proof: We prove the lemma by contradiction. Let $f=$ $\left(f_{1}, \cdots, f_{n}\right)$ be any optimal traffic partition satisfying that $f_{i} \leq$ 2 for all $1 \leq i \leq n$ and the cardinality of the set

$$
\left\{1 \leq i \leq n \mid f_{i}>r_{i} \bmod 4\right\}
$$

is the smallest. Assume that $f_{i} \leq r_{i} \bmod 4$ for some node $i$. Then in a canonical optimal grooming of the traffic carried in high-speed rings, in addition to $\left\lfloor\frac{r_{i}-f_{i}}{4}\right\rfloor$ high-speed rings which are devoted exclusively to node $i$, one high-speed ring carries the remaining $4-f_{i}+r_{i} \bmod 4$ amount of traffic from node $i$. This high-speed ring must also carry traffic from other nodes, for otherwise we can we fill this ring fully with the traffic from node $i$ without any additional cost but the amount of traffic placed in low-speed rings is $r_{i} \bmod 4$, which contradicts to the selection of $f=\left(f_{1}, \cdots, f_{n}\right)$. Let $x_{i}>0$ be the amount of the traffic carried in this ring from nodes other than node $i$. Then $x_{i}>1$ for 
otherwise we can decrease the total ADM cost by 0.5 by moving $x_{i}$ to a dedicated low-speed ring, which again contradicts to the optimality of $f=\left(f_{1}, \cdots, f_{n}\right)$. As

$$
4-f_{i}+r_{i} \bmod 4+x_{i} \leq 4
$$

we have

$$
1<x_{i} \leq r_{i} \bmod 4+x_{i} \leq f_{i} \leq 2
$$

This implies that $x_{i}$ is from only one node, say $j$, for otherwise the portion of the traffic from some node is less than one and again we can decrease the total ADM cost by the moving of it to a dedicated low-speed ring. Now we look at the $f_{i}$ amount of traffic from node $i$ carried in low-speed rings. In a canonical optimal grooming, one ring carries the traffic of amount 1 from node $i$ only, another ring carries $f_{i}-1$ amount of traffic from node $i$ and may carry additional traffic from other nodes. Finally we relocate all traffic in these three rings as follows. Fill the high-speed ring fully with the traffic from node $i$. Fill the first low-speed ring fully with the traffic from node $j$. In the second low-speed ring, keep the original traffic not from node $i$, and place $r_{i} \bmod 4$ amount of traffic from node $i$ and $x_{i}-1$ amount of traffic from node $j$. With this modification, one highspeed ADM is saved but one additional low-speed ADM is used. So the total cost is decreased by $2.5-1=1.5$, which again contradicts to the optimality of $f=\left(f_{1}, \cdots, f_{n}\right)$.

From the above lemma, there is an optimal solution in which $\left\lfloor\frac{r_{i}}{4}\right\rfloor$ high-speed rings are dedicated $r_{i}-r_{i} \bmod 4$ amount of traffic from node $i$ for all $1 \leq i \leq n$. Thus from now on, we assume that $r_{i}<4$ for all node $i$. For any traffic partition $f=\left(f_{1}, \cdots, f_{n}\right)$, let

$$
\begin{gathered}
S(f)=\left\{1 \leq i \leq n \mid 0<f_{i}<r_{i}\right\}, \\
U(f)=\left\{1 \leq i \leq n \mid f_{i}=0 \text { or } r_{i}\right\} .
\end{gathered}
$$

Thus the traffic from any node in $S(f)$ is carried in both lowspeed rings and high-speed rings, and the traffic from any node in $U(f)$ is carried in either low-speed rings or high-speed rings but not both.

The next lemma states that at any node, if the traffic of this node is carried in both types of rings, then the amount of traffic carried in low-speed rings is at most one; and if there is some traffic carried in a high-speed ring, its amount is more than one.

Lemma 5: Let $f=\left(f_{1}, \cdots, f_{n}\right)$ be any optimal traffic partition. Then for any $1 \leq i \leq n$, neither $1<f_{i}<r_{i}$ nor $0<r_{i}-f_{i} \leq 1$ is possible.

Proof: Assume that $1<f_{i}<r_{i}$. Then in a canonical optimal grooming, the total cost of ADMs used by the traffic $r_{i}$ is at least

$$
2+1+2.5=5.5
$$

as at least 2 low-speed ADMs is needed at node $i$, at least 1 lowspeed ADM is needed at the hub, and at least 1 high-speed ADM is required at the node $i$. But if the traffic $r_{i}$ is entirely carried by a high-speed ring, the cost of ADMs is at most $2.5+2.5=5<$
5.5, which contradicts to the optimality of $f=\left(f_{1}, \cdots, f_{n}\right)$. Now we assume that $0<r_{i}-f_{i} \leq 1$. We remove the $r_{i}-$ $f_{i}$ amount of traffic from the high-speed ring and put it in a dedicated low-speed ring. With this modification, at least one high-speed ring is saved and two additional low-speed ADMs are used. So the total cost is decreased by

$$
2.5-2=0.5
$$

which again is impossible as $f=\left(f_{1}, \cdots, f_{n}\right)$ is already optimal.

As a corollary of Lemma 5, in any canonical optimal grooming, any high-speed ring can carry traffic from at most three nodes.

The next lemma states that, at any node, when a traffic demand from a node is at most one, it should be always put in a low-speed ring; and when a traffic demand is more than three, it should be always put in a high-speed ring.

Lemma 6: Let $f=\left(f_{1}, \cdots, f_{n}\right)$ be any optimal traffic partition. Then for any $1 \leq i \leq n$, if $r_{i} \leq 1, f_{i}=r_{i}$; and if $r_{i}>3$, $f_{i}=0$.

Proof: The first part follows directly from Lemma 5. Now we assume that $r_{i}>3$ and $f_{i}>0$. From Lemma 3 and Lemma $5,0<f_{i} \leq 1$, and thus $r_{i}-f_{i}>2$. The $r_{i}-f_{i}$ amount of traffic from node $i$ must share some traffic from other nodes, for otherwise we can put all traffic from node $i$ in the high-speed ring and decreases the cost by at least one. From Lemma 5 if there is some traffic, from any node, carried in a high-speed ring, its amount is more than one. Thus the $r_{i}-f_{i}$ amount of traffic from node $i$ share one high-speed ring with some amount, denoted by $x_{i}$, of traffic from exactly one node, say $j$. Note that

$$
1<x_{i} \leq 4-r_{i}+f_{i}
$$

So we consider the following modification to a canonical optimal solution. We replace the $f_{i}$ amount of traffic from node $i$ in some low-speed ring by the $f_{i}$ amount of traffic from node $j$. This may save one low-speed ADM. We then place the $x_{i}-f_{i}$ in a dedicated low-speed ring as

$$
x_{i}-f_{i} \leq 4-r_{i}<1
$$

This adds two low-speed ADMs. Finally, we place all traffic from node $i$ in the high-speed ring originally carrying the $r_{i}-f_{i}$ amount of traffic from node $i$ and $x_{i}$ amount of traffic from node $j$. This saves one high-speed ADM. Thus after the modification, the total ADM cost is decreased by at least $2.5-2=0.5$, which contradicts to the optimality of $f=\left(f_{1}, \cdots, f_{n}\right)$.

The above lemma implies that if $r_{i} \leq 1$ for any node $1 \leq$ $i \leq n$, then all traffic must be carried in low-speed rings. In particular, if the traffic is uniform with amount $r$, the total ADM cost is $F(1, r, n)$. If $r_{i}>3$ for any node $1 \leq i \leq n$, then all traffic must be carried in high-speed rings. As in the canonical grooming, the traffic demand from any node must be carried in a dedicated high-speed ring. Thus $2 n$ high-speed ADMs are 
needed with cost $5 n$ in total. A remark is such cost only accounts for the working ring, if we consider the protection as well, the total cost should then be doubled.

\section{All Traffic Demands Are at Most Two}

In the next lemma, we show that when the traffic demand from each node is at most two, then there is an optimal traffic partition in which none of them is carried in both low-speed rings and high-speed rings.

Lemma 7: If $r_{i} \leq 2$ for all $1 \leq i \leq n$, then there is an optimal traffic partition $f$ with $S(f)=\emptyset$.

Proof: We prove it by contradiction. Let $f=\left(f_{1}, \cdots, f_{n}\right)$ be any optimal traffic partition with the smallest $|S(f)|$. Let $i \in S(f)$ and consider any canonical optimal grooming. From Lemma $5,0<f_{i} \leq 1$ and $r_{i}-f_{i}>1$. Thus in any canonical optimal grooming, the traffic from node $i$ is carried in exactly one low-speed ring and exactly one high-speed ring. We concentrate on the high-speed ring carrying the $r_{i}-f_{i}$ amount of traffic from node $i$. It can carry traffic from at most three nodes. First of all, it must also carry some traffic from other nodes, for otherwise we can fill it with all traffic from node $i$ and decreases the cost by at least one. Secondly, it is impossible that this highspeed ring carries the traffic from only two nodes, for otherwise we can put all traffic from these two nodes in this high-speed ring, which can also save at least one low-speed ADM. Thus this high-speed ring must carry traffic from exactly three nodes. We denote the other two nodes other than node $i$ by $j$ and $k$. We show that $j, k \in U(f)$. Suppose to the contrary. We modify the placement of the traffic from these three nodes as follows. We use the high-speed ring to carry the whole traffic from node $i$ and the whole traffic from node $j$ and nothing else. We add at most two new dedicated low-speed rings to carry the traffic from node $k$. We save one high-speed ADM and add at most two more low-speed ADMs. Thus the modification decreases the total cost by at least 0.5 , which contradicts to the optimality of $f$. Therefore both $j$ and $k$ are in $U(f)$, that is all traffic from node $j$ and node $k$ are carried in the high-speed ring. As $r_{i}-f_{i}>1$

$$
r_{j}+r_{k} \leq 4-\left(r_{i}-f_{i}\right)<4-1=3
$$

So we can modify the placement of the traffic from nodes $i, j$ and $k$ as follows. We place all the traffic from node $i$ and nothing else in two new low-speed rings, and use at most three new lowspeed rings to carry all traffic from nodes $j$ and $k$. Then four high-speed ADMs are saved, and at most ten low-speed ADMs are added. The resulting solution has the same cost as $f$ but it contains one less nodes whose traffic are carried in both lowspeed rings and high-speed rings. This contradicts to that $|S(f)|$ is the smallest. Thus the lemma is true.

\section{A. All Traffic Demands Are at Most $\frac{3}{2}$}

The next lemma states that when the traffic demand from each node is at most $\frac{3}{2}$, then we can put all traffic in the low-speed rings.

Lemma 8: If $r_{i} \leq \frac{3}{2}$ for all $1 \leq i \leq n$, then the traffic partition $f=\left(f_{1}, \cdots, f_{n}\right)$ where $f_{i}=\bar{r}_{i}$ for all $1 \leq i \leq n$ is optimal.

Proof: We prove it by contradiction. Let $f=\left(f_{1}, \cdots, f_{n}\right)$ be any optimal traffic partition with $f_{i}=0$ or $r_{i}$ for all $1 \leq i \leq n$ and the smallest number of zero entries. Consider any canonical optimal grooming. As any high-speed ring carries traffic from at most three nodes. We consider the following three cases. If a high-speed ring carries traffic from only one node, we can use at most two new low-speed rings to carry all traffic from this node. This modification saves two highspeed ADMs and uses at most four low-speed ADMs. Thus the cost is decreased by 0.5 , which contradicts to the optimality of $f=\left(f_{1}, \cdots, f_{n}\right)$. If a high-speed ring carries traffic from two nodes, we can use at most three new low-speed rings to carry all traffic from these nodes. This modification saves three highspeed ADMs and uses at most seven low-speed ADMs. Thus the cost is decreased by 0.5 , which also contradicts to the optimality of $f=\left(f_{1}, \cdots, f_{n}\right)$. If a high-speed ring carries traffic from three nodes, we use at most four new low-speed rings to carry all traffic in this high-speed ring. This modification saves four high-speed ADMs and uses at most ten low-speed ADMs. The resulting solution has the same cost as $f$, but the number of zero entries is decreased by three, which contradicts to the selection of $f$. Therefore, the lemma is true.

The above lemma implies if the traffic is uniform with demand $r \leq \frac{3}{2}$, the minimum cost of ADMs is $F(1, r, n)$.

\section{B. All Traffic Demands Are More than $\frac{3}{2}$}

We now consider the traffic with demands more than $\frac{3}{2}$ but at most two.

Lemma 9: Suppose that $\frac{3}{2}<r_{i} \leq 2$ for all $1 \leq i \leq n$. If $n$ is even, then the traffic partition $f=\left(f_{1}, \cdots, f_{n}\right)$ where $f_{i}=0$ for all $1 \leq i \leq n$ is optimal. If $n$ is odd, then for any $1 \leq j \leq n$ the traffic partition $f=\left(f_{1}, \cdots, f_{n}\right)$ where $f_{i}=0$ for $i \neq j$ and $f_{j}=r_{j}$ is optimal.

Proof: We also prove it by contradiction that there is an optimal traffic partition $f=\left(f_{1}, \cdots, f_{n}\right)$ with $f_{i}=0$ or $r_{i}$ for all $1 \leq i \leq n$ and at most one non-zero entries. Let $f=\left(f_{1}, \cdots, f_{n}\right)$ be any optimal traffic partition with $f_{i}=0$ or $r_{i}$ for all $1 \leq i \leq n$ and the smallest number of non-zero entries. Assume that $f_{i}=r_{i}$ and $f_{j}=r_{j}$. Consider any canonical optimal grooming. There are two low-speed rings devoted to node $i$ and two low-speed rings devoted to node $j$. We relocate the traffic from node $i$ and node $j$ to one new high-speed ring. This modification saves 8 low-speed ADMs and uses 3 
high-speed ADMs. The total cost is decreased by 0.5 . This contradicts to the optimality of $f=\left(f_{1}, \cdots, f_{n}\right)$. Now let $f=\left(f_{1}, \cdots, f_{n}\right)$ be any optimal traffic partition with $f_{i}=0$ or $r_{i}$ for all $1 \leq i \leq n$ and at most one non-zero entries. Note that in any canonical optimal grooming, each high-speed ring must carry traffic from two nodes, for otherwise we can move it to two low-speed rings and the cost would be decreased by 1 . Thus if $n$ is even, $f_{i}=0$ for all $1 \leq i \leq n$, and if $n$ is odd, there is exactly one $1 \leq i \leq n$ with $f_{i}=r_{i}$.

From the above lemma, if $\frac{3}{2}<r_{i} \leq 2$ for all $1 \leq i \leq n$ we can provide optimal grooming as follows. If $n$ is even, then all traffic is carried in high-speed rings, and each high-speed ring carries the whole traffic from two nodes. It requires totally $1.5 n$ high-speed ADMs (in the working ring only) with total cost $3.75 n$. If $n$ is odd, then the traffic from one node is carried in two low-speed rings to carry the whole traffic from a node, and the traffic from all other nodes are carried in the high-speed rings, with each ring dedicated to a pair of nodes. Thus 4 lowspeed ADMs and 1.5 $(n-1)$ high-speed ADMs are used. So the total ADM cost is

$$
4+1.5(n-1) \cdot 2.5=3.75 n+1.5 .
$$

\section{All Traffic Demands Are More than Two}

In general, each high-speed ring can carry traffic from at most three nodes. The next lemma states that if all traffic demands are more than two, then in any canonical optimal grooming no highspeed ring can carry traffic from three nodes.

Lemma 10: If $r_{i}>2$ for all node $i$, then in any canonical optimal grooming each high-speed ring carries traffic from at most two nodes.

Proof: We prove it by contradiction. Consider a canonical optimal grooming with traffic partition $f=\left(f_{1}, \cdots, f_{n}\right)$. Assume that three nodes $i, j$ and $k$ appear in a high-speed ring. Then $i, j, k \in S(f)$ for otherwise

$$
\left(r_{i}-f_{i}\right)+\left(r_{j}-f_{j}\right)+\left(r_{k}\right.
$$

As

$$
\left(r_{i}-f_{i}\right)+\left(r_{j}-f_{j}\right)+\left(r_{k}\right.
$$

we have

$$
f_{i}+f_{j}+f_{k} \geq r_{i}+r_{j}+r_{k}-4>2 .
$$

As $f_{k} \leq 1, f_{i}+f_{j}>1$, so are $f_{i}+f_{k}$ and $f_{j}+f_{k}$. This means that all the three nodes must appear in three distinct low-speed rings. Assume these three rings carry $x_{i}, x_{j}$ and $x_{k}$ amount of the traffic from other nodes respectively. Then we have

$$
x_{i}+x_{j}+x_{k} \leq 3-\left(f_{i}+f_{j}+f_{k}\right)<1 .
$$

Note that

$$
r_{i}+r_{j}+r_{k} \leq f_{i}+f_{j}+f_{k}+4 \leq 7,
$$

As $r_{k}>2, r_{i}+r_{j}<5$, so are $r_{i}+r_{k}$ and $r_{j}+r_{k}$. Now we relocate the traffic carried in these three low-speed rings and the high-speed ring as follows. We place the whole traffic from node $i$ in the high-speed ring, place the whole traffic from node $j$ and $4-r_{j}$ amount of traffic from node $k$ in a new high-speed ring, and place $r_{j}+r_{k}-4$ amount of traffic from node $k$ in a low-speed ring as

$$
0<r_{j}+r_{k}-4<1
$$

The $x_{i}, x_{j}$ and $x_{k}$ amount of the traffic from other nodes are carried exclusively in another low-speed ring. After the relocation, we save three low-speed ADMs and add one high-speed ADM. So the total cost is decreased by 0.5 , which is a contradiction.

The following lemma states that if all traffic demands are greater than two, we can concentrate on those canonical grooming in which exactly one node in each high-speed ring has its whole traffic carried in this high-speed ring.

Lemma 11: If $r_{i}>2$ for all $1 \leq i \leq n$, then there is a canonical optimal grooming in which exactly one node in each high-speed ring has its whole traffic carried in this high-speed ring.

Proof: We prove it by contradiction. Consider a canonical optimal grooming with traffic partition $f=\left(f_{1}, \cdots, f_{n}\right)$ with $f_{i} \leq 2$ for all $1 \leq i \leq n$. From Lemma $5, f_{i} \leq 1$ for all $1 \leq i \leq n$. Thus for all $1 \leq i \leq n$,

$$
r_{i}-f_{i}>2-1=1 \text {. }
$$

If a high-speed carries traffic from only one node, then it must carry the whole traffic from that node. Now we consider a highspeed ring which carries traffic from two nodes $i, j \in S(f)$. We relocate the traffic from node $i$ and node $j$ as follows. The high-speed ring carries $r_{i}$ amount of traffic from node $i$, and $4-r_{i}$ amount of traffic from node $j$. We replace the original $f_{i}$ amount of traffic from node $i$ in a low-speed ring by $f_{i}$ amount of traffic from node $j$. The cost of the result grooming is not increased. We repeat such procedure for all high-speed rings which each carry traffic from two nodes that are both in $S(f)$. In the end, we come up with a grooming in which each high-speed ring carries the whole traffic from at least one node. Finally we use a canonical grooming to place all traffic carried in low-speed rings. Then the resulting grooming satisfies the requirement given in the lemma.

\section{A. All Traffic Demands Are More than $\frac{5}{2}$}

When all traffic demands are greater than $\frac{5}{2}$, the following lemma gives an optimal traffic partition.

Lemma 12: If $r_{i}>\frac{5}{2}$ for all $1 \leq i \leq n$, then the traffic partition $f=\left(f_{1}, \cdots, f_{n}\right)$ where $f_{i}=0$ for all $1 \leq i \leq n$ is optimal. 
Proof: We consider a canonical optimal grooming with the traffic partition $f=\left(f_{1}, \cdots, f_{n}\right)$ in which each high-speed ring carries the whole traffic from at least one node. Assume that $f_{i}>0$ for some $1 \leq i \leq n$. From Lemma $5, f_{i} \leq 1$. Furthermore, the high-speed ring where node $i$ appears must carry the whole traffic from another node, say $j$, and no other traffic. As

$$
\left(r_{i}-f_{i}\right)+r_{j} \leq 4
$$

we have

$$
f_{i} \geq r_{i}+r_{j}-4>1 .
$$

This contradicts to $f_{i} \leq 1$.

The above lemma suggests that if all traffic demands are more than $\frac{5}{2}$, we should carry all traffic in high-speed rings. In this optimal traffic partition, the canonical grooming is unique and each high-speed ring carries exclusively the whole traffic from only one node. Thus the minimal total ADM cost (in the working ring) is $5 n$.

\section{B. All Traffic Demands Are at Most $\frac{5}{2}$}

Finally we consider the traffic with demands at most $\frac{5}{2}$ but more than two. The next lemma states that if all traffic demands are at most $\frac{5}{2}$, then in any optimal grooming there is at most one high-speed ring which carries exclusively the whole traffic from exactly one node.

Lemma 13: If $r_{i} \leq \frac{5}{2}$ for all $1 \leq i \leq n$, then in any optimal grooming at most one high-speed ring carries exclusively the whole traffic from exactly one node.

Proof: We prove it by contradiction. Consider an optimal grooming with traffic partition $f=\left(f_{1}, \cdots, f_{n}\right)$ in which there are two high-speed ring dedicated to node $i$ and node $j$ repulsively. We relocate the traffic from node $i$ and node $j$ as follows. We place $r_{i}$ amount of traffic from node $i$, and $\min \left\{4-r_{i}, r_{j}\right\}$ amount of traffic from node $j$ on one high-speed ring, and if $r_{i}+r_{j}>4$ we place $r_{i}+r_{j}-4$ amount of traffic from node $j$ on one low-speed ring. This modification saves one high-speed ADM and adds at most two low-speed ADMs. The cost is decreased by at least 0.5 , which is a contradiction.

From Lemma 10, 11 and 13, if $n$ is even and $2<r_{i} \leq \frac{5}{2}$ for all $1 \leq i \leq n$, then there is a canonical optimal grooming in which half nodes have their traffic carried in high-speed rings and the half node have their traffic carried in both high-speed rings and low-speed ring, and each high-speed is fully filled with the whole traffic from one node in the first half and a portion of traffic from a node in the second half. if $n$ is odd and $2<r_{i} \leq \frac{5}{2}$ for all $1 \leq i \leq n$, then there is a canonical optimal grooming in which the traffic from one node is carried exclusively in a high-speed ring and the traffic from other nodes are carried in the same way as the number of nodes is even. However, how to select the set of nodes to be carried wholly in high-speed rings and how to form node pairs to appear in high-speed rings remains open. But if the traffic is uniform, these two questions can be easily solved. We can select any $\left\lceil\frac{n}{2}\right\rceil$ nodes to be carried wholly in high-speed rings, and the pairing between those nodes and the remaining nodes can be selected arbitrarily. Thus, for uniform traffic with demand $2<r \leq \frac{5}{2}$, the total ADM cost in the working ring is

$$
3.25 n+F\left(1,2 r-4, \frac{n}{2}\right)
$$

if $n$ is even, and is

$$
\begin{gathered}
5+3.25(n-1)+F\left(1,2 r-4, \frac{n-1}{2}\right) \\
=1.75+3.25 n+F\left(1,2 r-4, \frac{n-1}{2}\right)
\end{gathered}
$$

if $n$ is odd.

\section{SUMMARY}

For uniform traffic demands, we have provided optimal traffic partition and grooming, which is summarized in Table I. For non-uniform traffic demands, optimal or suboptimal solutions have been developed depending on the range of all demands. If all demands are at most 1.5, then all of them are carried in lowspeed rings. If all traffic demands are greater than 1.5 but less than two, then with even $n$, all of them are carried in high-speed rings and the total cost of ADMs in the working ring only is $3.75 n$; with odd $n$, all of them except an arbitrary one are carried in high-speed rings and the total cost of ADMs in the working ring only is $3.75 n+1.5$. Such costs remain the same as long as all demands are greater than 1.5 but less than two. If all traffic demands are greater than 2.5 , all of them are carried in highspeed rings and the total cost of ADMs in the working ring only is $5 n$. Such cost also remain the same as long as all demands are greater than 2.5. When all traffic demands are greater than two but less than 2.5, the solution is a little complicated. We first pair up the $n$ nodes. If $n$ is odd, some node is stand-alone and its whole traffic is carried in a high-speed ring. For each pair of nodes $i$ and $j$, we use a high-speed ring to carry the whole traffic from node $i$ and the remaining capacity is used to carry the traffic from node $j$.

TABLE I

SELECT LINE SPEEDS FOR UPSR

\begin{tabular}{|c|c|}
\hline Range of all $r$ 's & $\left(f_{1}, f_{2}, \cdots, f_{n}\right)$ \\
\hline$\left(0,1 \frac{1}{2}\right]$ & $f_{i}=r, \forall i$ \\
\hline$\left(1 \frac{1}{2}, 2\right], n=2 k$ & $f_{i}=0, \forall i$ \\
\hline$\left(1 \frac{1}{2}, 2\right], n=2 k+1$ & $f_{i}=0, \forall i \neq j ; f_{j}=r$ \\
\hline$\left(2,2 \frac{1}{2}\right]$ & $f_{2 i-1}=0, f_{2 i}=2 r-4$ \\
\hline$\left(2 \frac{1}{2}, 4\right]$ & $f_{i}=0, \forall i$ \\
\hline
\end{tabular}

The above argument is restricted to UPSR. However, it can be extended to BLSR as well. Table II lists the optimal traffic partition of uniform traffic demands. 
TABLE II

SELECT LINE SPEEDS FOR BLSR/2

\begin{tabular}{|c|c|}
\hline Range of all $r$ 's & $\left(f_{1}, f_{2}, \cdots, f_{n}\right)$ \\
\hline \hline$\left(0, \frac{3}{4}\right]$ & $f_{i}=r, \forall i$ \\
\hline$\left(\frac{3}{4}, 1\right], n=2 k$ & $f_{i}=0, \forall i$ \\
\hline$\left(\frac{3}{4}, 1\right], n=2 k+1$ & $f_{i}=0, \forall i \neq j ; f_{j}=r$ \\
\hline$\left(1,1 \frac{1}{4}\right]$ & $f_{2 i-1}=0, f_{2 i}=2 r-2$ \\
\hline$\left(1 \frac{1}{4}, 2\right]$ & $f_{i}=0, \forall i$ \\
\hline
\end{tabular}

\section{REFERENCES}

[1] E.G. Coffman, Jr. and M.R. Garey and D.S. Johnson, "Approximation Algorithms for Bin-Packing - An Updated Survey", Algorithm Design for Computer System Design, 1984.

[2] C. Colbourn, and P.-J. Wan, "Minimizing Drop Cost for SONET/WDM Networks with $\frac{1}{8}$ Wavelength Requirements", in preparation.

[3] O. Gerstel, P. Lin, and G. Sasaki, "Cost effective traffic grooming in WDM rings", Proc. IEEE Infocom 98, vol. 1 pp. 69 -77.

[4] O. Gerstel, P. Lin, and G. Sasaki, "Wavelength assignment in a WDM ring to minimize cost of embedded SONET rings", Proc. IEEE Infocom 98, vol. 1 pp. $94-101$.

[5] O. Gerstel, P. Lin, and G. Sasaki, "Combined WDM and SONET Network Design", Proc. IEEE Infocom 99.

[6] I. Haque, W. Kremer, and K. Raychauduri, Self-Healing Rings in a synchronous environment, SONET/SDH: a sourcebook of synchronous networking, Eds. C.A. Siller and M. Shafi, IEEE Press, New York, pp. 131139, 1996.

[7] L.W. Liu, P.-J. Wan, and O. Frieder, "Wavelength Assignment in WDM Rings to Minimize SONET ADMs", submitted for publication, 1999.

[8] E. Modiano and A. Chiu, "Traffic Grooming Algorithms for Minimizing Electronic Multiplexing Costs in Unidirectional SONET/WDM Networks", CISS '98, Princeton, NJ, March, 1998.

[9] B. Mukherjee, Optical Communication Networks, New York: McGrawHill, July 1997.

[10] J. Simmons, E. Golstein, and A. Saleh, "Quantifying the Benefit of Wavelength Add-Drop in WDM Rings with Distance-Independent and Dependent Traffic", Journal of Lightwave Technology, Vol. 17, No. 1, January 1999, pp. 48-57.

[11] P.-J. Wan, and C. Colbourn, "Combinatorial Design of Bidirectional SONET/WDM Rings with $\frac{1}{2}$ and $\frac{1}{4}$ Wavelength Uniform Traffic", submitted for publication, 1999 .

[12] P.-J. Wan, X.-Y. Li, and L.-W. Liu "Traffic Grooming in Single-Hub SONET/WDM Ring Networks", submitted for publication, 1999.

[13] G. T. Wilfong., "Minimizing wavelengths in an all-optical ring network", Proceedings of 7th International Symposium on Algorithms and Computation, pages 346-355, 1996.

[14] A. C. Yao, "New Algorithms in Bin Packing", technical report, Computer Science Department, Stanford, 1978. 